\title{
A SIMULTANEOUS SOLUTION TO TWO PROBLEMS ON DERIVATIVES
}

\section{F.S. CATER}

Department of Mathematics Portland State University Portland, Oregon 97207

(Received March 7, 1985)

ABSTRACT. Let A be a nonvoid countable subset of the unit interval $[0,1]$ and let $B$ be an $F_{\sigma}$-subset of $[0,1]$ disjoint from $A$. Then there exists a derivative $f$ on $[0,1]$ such that $0 \leq f \leq 1, f=0$ on $A, f>0$ on $B$, and such that the extended real valued function $1 / f$ is also a derivative.

KEY WORDS AND PHRASES. Derivative, primitive, Lebesgue summable, knot point. 1980 MATHEMATICS SUBJECT CLASSIFICATION CODE. 26A24

In this note, we construct a derivative $f$ such that $1 / \mathrm{f}$ is also a derivative, and $f$ and $1 / f$ have some curious properties mentioned in [1] and [2]. (By an $F_{\sigma}$-set in the real line, we mean the union of countably many closed subsets of $R$. ) We prove

THEOREM 1. Let $A$ be a nonvoid countable subset of $[0,1]$ and let $B$ be an $F_{\sigma}-$ subset of $[0,1]$ disjoint from $A$. Then there exists a measurable function $f$ on $[0,1]$ such that $f=0$ on $A, f>0$ on $B, 0 \leqq f \leqq 1$ on $[0,1]$ and

(1) $f$ is everywhere the derivative of its primitive,

(2) $1 / \mathrm{f}$ is Lebesgue summable on $[0,1]$,

(3) $1 / \mathrm{f}$ is everywhere the derivative of its primitive.

Here we let $\infty=1 / 0$.

When $m(B)=1$ and $A$ is dense, we will obtain a simple example of a derivative that vanishes on a dense set of measure 0 .

From [2] we infer that there exists a derivative $f$ vanishing on $A$ and positive on B. From [1] we infer that there exists a derivative $g$ infinite on $A$ and $f$ inite on $B$. However, Theorem 1 provides a simultaneous solution to both of these problems. To prove Theorem 1 we will employ some of the methods used in [3].

Finally, we use these methods to construct a concrete example of functions $g_{1}$ and $g_{2}$ that have finite or infinite derivatives at each point, such that the Dini derivatives of their difference, $g_{1}-g_{2}$, satisfy certain pathological properties.

In all that follows, let $(\mathrm{n}(\mathrm{i}))_{i=1}^{\infty}$ denote the sequence of integers $1,1,2,1,2$, $3,1,2,3,4,1,2,3,4,5,1, \ldots$.

Proof of Theorem 1. Let $\left(a_{i}\right)_{i=1}^{\infty}$ be a sequence of points in A such that each point of $A$ occurs at least once in the sequence. (Here we do not exclude the possibility that $\mathrm{A}$ is a finite set.) We assume, without loss of generality, that $\mathrm{B}$ is nonvoid. 
Let $B_{1} \subset B_{2} \subset B_{3} \subset \ldots \subset B_{i} \ldots$ be an expanding sequence of closed sets such that $B={ }^{\infty}{ }_{i=1}^{\infty} B_{i}$. (Here we do not exclude the possibility that $B$ is a closed set.) Let $u_{i}$ denote the distance from the point $a_{n(i)}$ to the set $B_{i}$. As in [3], we put $\emptyset(x)=(1+|x|)^{-\frac{1}{2}}$. For each index $j$, put

$$
\begin{aligned}
& g_{j}(x)=1+\sum_{i=1}^{j} \emptyset\left(2^{i} u_{i}^{-1}\left(x-a_{n(i)}\right)\right), \\
& g(x)=1+\sum_{i=1}^{\infty} \emptyset\left(2^{i} u_{i}^{-1}\left(x-a_{n(i)}\right)\right), \\
& f_{j}(x)=1 / g_{j}(x), \quad f(x)=1 / g(x) .
\end{aligned}
$$

Here we let $0=1 / \infty$. Then $g(a)=\infty$ for a $\varepsilon A$, because there are infinitely many indices $i$ for which $a=a_{n(i)}$. On the other hand, $g(b)<\infty$ for $b \varepsilon B$; note that if $b \varepsilon B_{k}$, then

$$
\begin{gathered}
\emptyset\left(2^{k} u_{k}^{-1}\left(b-a_{n(k)}\right)\right) \leqq \emptyset\left(2^{k}\right)<2^{-\frac{1}{2} k}, \\
\sum_{i=k}^{\infty} \emptyset\left(2^{i} u_{i}^{-1}\left(b-a_{n(i)}\right)\right) \leqq \sum_{i=k}^{\infty} 2^{-\frac{1}{2} i}<\infty .
\end{gathered}
$$

We infer from Lemma 4 of [3], that $g$ is Lebesgue summable on $[0,1]$. Note also that

$$
g(x)-g_{j}(x)=g(x) g_{j}(x)\left(f_{j}(x)-f(x)\right)>0,
$$

and since $g>1, g_{j}>1$, it follows that $g-g_{j}>f_{j}-f>0$.

Now choose any $x$ with $g(x)<\infty$. By Lemma 4 of [3], we have

$$
\lim _{h \rightarrow 0} h^{-1} \int_{x}^{x+h} g(t) d t=g(x) \text {. }
$$

Take any $\varepsilon>0$. Select an index $j$ so large that $f_{j}(x)-f(x)<g(x)-g_{j}(x)<\varepsilon$. Since $f_{j}$ and $g_{j}$ are continuous, when $|h|$ is small enough we have

$$
\begin{aligned}
& \left|h^{-1} \int_{x}^{x+h} g(t) d t-g(x)\right|<\varepsilon, \\
& \left|h^{-1} \int_{x}^{x+h} g_{j}(t) d t-g_{j}(x)\right|<\varepsilon, \\
& \left|h^{-1} \int_{x}^{x+h} f_{j}(t) d t-f_{j}(x)\right|<\varepsilon .
\end{aligned}
$$

For such $j$ and $h$ we obtain

$$
\begin{aligned}
h^{-1} \int_{x}^{x+h}\left(g(t)-g_{j}(t)\right) d t \leq g(x)-g_{j}(x) & +\left|h^{-1} \int_{x}^{x+h} g(t) d t-g(x)\right| \\
& +\left|h^{-1} \int_{x}^{x+h} g_{j}(t) d t-g_{j}(x)\right|<3 \varepsilon .
\end{aligned}
$$

From $0<\mathrm{f}_{\mathrm{j}}-\mathrm{f}<\mathrm{g}-\mathrm{g}_{\mathrm{j}}$ we obtain

$$
\begin{aligned}
\left|h^{-1} \int_{x}^{x+h} f(t) d t-f(x)\right| & \leqq\left|h^{-1} \int_{x}^{x+h} f_{j}(t) d t-f_{j}(x)\right|+f_{j}(x)-f(x) \\
& +h^{-1} \int_{x}^{x+h}\left(f_{j}(t)-f(t)\right) d t \\
& \leqq 2 \varepsilon+h^{-1} \int_{x}^{x+h}\left(f_{j}(t)-f(t)\right) d t \\
& \leqq 2 \varepsilon+h^{-1} \int_{x}^{x+h}\left(g(t)-g_{j}(t)\right) d t<5 \varepsilon .
\end{aligned}
$$

It follows that $\lim _{h \rightarrow 0} h^{-1} \int_{x}^{x+h} f(t) d t=f(x)$. 
Choose any $x$ with $g(x)=\infty$. Take any $N>0$. Select $j$ so large that $g_{j}(x)>N$. Since $g_{j}$ is continuous, there is a $d>0$ such that $|t-x|<d$ implies $g_{j}(t)>N$. For such $t, g(t)>g_{j}(t)>N$ and $f(t)<f_{j}(t)<N^{-1}$. It follows that for $|h|<d$,

$$
h^{-1} \int_{x}^{x+h} g(t) d t>N, 0<h^{-1} \int_{x}^{x+h} f(t) d t<N^{-1} .
$$

Fina11y,

$$
\begin{aligned}
& \lim _{h \rightarrow 0} h^{-1} \int_{x}^{x+h} g(t) d t=\infty=g(x) . \\
& \lim _{h \rightarrow 0} h^{-1} \int_{x}^{x+h} f(t) d t=0=f(x) \text {. }
\end{aligned}
$$

This completes the proof.

When $m(B)=1$, we do not know if our argument can be modified to make $f=0$ on $[0,1] \backslash B$ as in [2]. Perhaps this requires an approach altogether different from ours. We say that $x$ is a knot point of the function $F$ if its Dini derivatives satisfy

$$
\mathrm{D}^{+} \mathrm{F}(\mathrm{x})=\mathrm{D}^{-} \mathrm{F}(\mathrm{x})=\infty \text { and } \mathrm{D}_{+} \mathrm{F}(\mathrm{x})=\mathrm{D}_{-} \mathrm{F}(\mathrm{x})=-\infty \text {. }
$$

We conclude by presenting a simple and direct example of functions $g_{1}$ and $g_{2}$ having derivatives (finite or infinite) at every point such that $g_{1}-g_{2}$ has knot points in every interval. (Consult [4] for analogous examples.)

Let $\left\{a_{i}\right\}_{i=1}^{\infty}$ and $\left\{b_{i}\right\}_{i=1}^{\infty}$ be countable dense subsets of $(0,1)$ that are disjoint. Let $Z(c, d, x)=\int_{0}^{x} \emptyset(c(t-d)) d t$ for $c>0, d>0, x>0$. We integrate to obtain

$$
Z(c, d, x)= \begin{cases}2 c^{-1}\left[(1+c d)^{\frac{1}{2}}-(1+c d-c x)^{\frac{1}{2}}\right] & \text { if } x \leqq d, \\ 2 c^{-1}\left[(1+c d)^{\frac{1}{2}}+(1+c x-c d)^{\frac{1}{2}}-2\right] & \text { if } x>d .\end{cases}
$$

Let $u_{i}$ denote the distance from $a_{n(i)}$ to the $\operatorname{set}\left\{b_{1}, \ldots, b_{i}\right\}$, and 1 let $v_{i}$ denote the distance from $b_{n(i)}$ to the set $\left\{a_{1}, \ldots, a_{i}\right\}$. Put

$$
g_{1}(x)=\sum_{i=1}^{\infty} z\left(2^{i} u_{i}^{-1}, a_{n(i)}, x\right), \quad g_{2}(x)=\sum_{i=1}^{\infty} z\left(2^{i} v_{i}^{-1}, b_{n(i)}, x\right)
$$

for $0<x<1$. By the argument in the proof of Theorem 1 we prove that $g_{1}$ and $g_{2}$ are absolutely continuous functions on $(0,1)$ with $g_{1}^{\prime}=\infty$ on $A, g_{2}^{\prime}=\infty$ on $B, g_{1}^{\prime}$ finite on $B$, and $g_{2}^{\prime}$ finite on $A$. Put $g=g_{1}-g_{2}$. Then $g$ is absolutely continuous on $(0,1)$, $\mathrm{g}^{\prime}=\infty$ on $\mathrm{A}$ and $\mathrm{g}^{\prime}=-\infty$ on $\mathrm{B}$. Each of the sets

$$
E_{1}=\left\{x: D^{+} g(x)=\infty\right\}, E_{2}=\left\{x: D^{-} g(x)=\infty\right\}, E_{3}=\left\{x: D_{+} g(x)=-\infty\right\} \text { and } E_{4}=\{x:
$$
$\left.D_{-} g(x)=-\infty\right\}$ is a dense $G_{\delta}$-subset of $(0,1)$, i.e., is the intersection of countably many open dense subsets of $(0,1)$. It follows that $E_{1} \cap E_{2} \cap E_{3} \cap E_{4}$ is also a dense $G_{\delta}$-subset of $(0,1)$. But each point in this intersection is a knot point of $g$, even though $g_{1}$ and $g_{2}$ have derivatives (finite or infinite) everywhere by the proof of Theorem 1 .

\section{REFERENCES}

1. CHOQUeT, G. Application des Propriétés Descriptives de la Functions Contingente à la Géométrie Différentielle des Variétés Cartésiennes, J. Math. Pures et App1. 26 (1947), 115-226.

2. ZAHORSKI, Z. Sur la Première Dérivée, Trans. Amer. Math. Soc. 69 (1950), 1-54, (Lemma 11).

3. KATZNELSON, Y. and STROMBERG, K. Everywhere Differentiable, Nowhere Monotone Functions, Amer. Nath. Monthly, 81 (1974), 349-354.

4. BELNA, C.L., CARGO, G.T., EVANS, M.J. and HUMKE, P.D. Analogues to the DenjoyYoung-Saks Theorem, Trans. Amer. Math. Soc. 271 (1982), 253-260. 


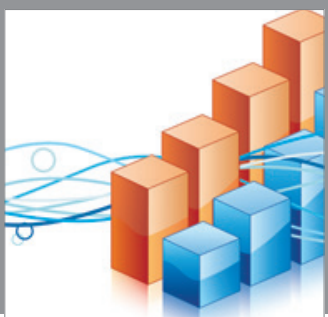

Advances in

Operations Research

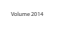

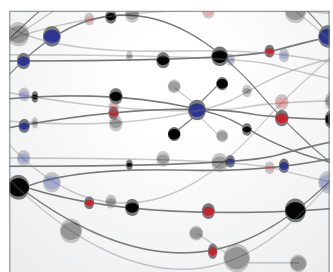

\section{The Scientific} World Journal
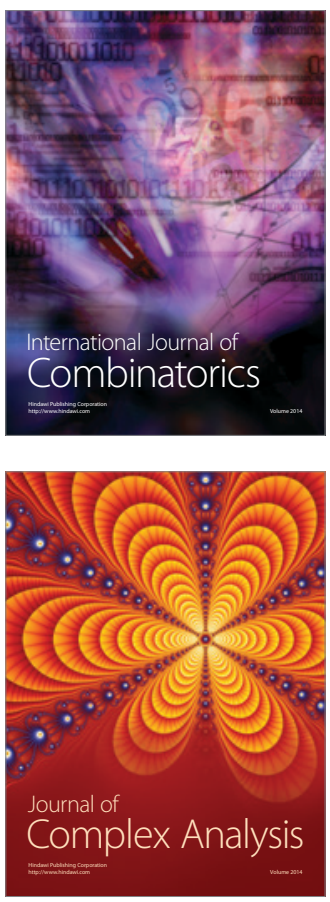

International Journal of

Mathematics and

Mathematical

Sciences
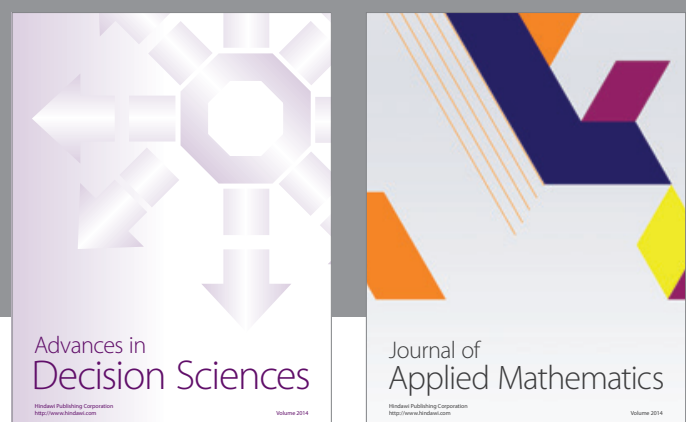

Journal of

Applied Mathematics
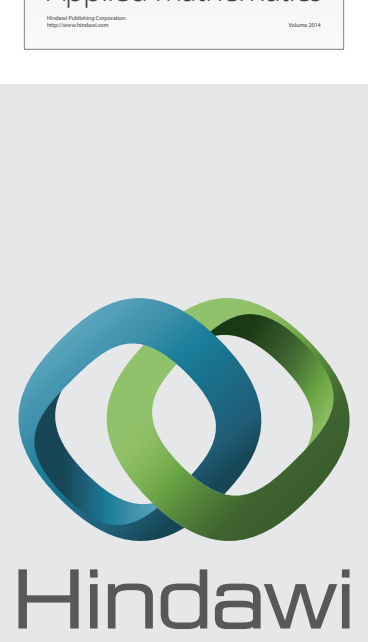

Submit your manuscripts at http://www.hindawi.com
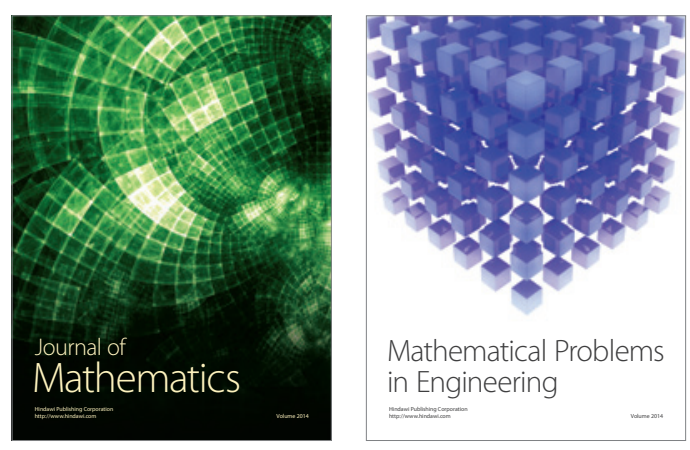

Mathematical Problems in Engineering
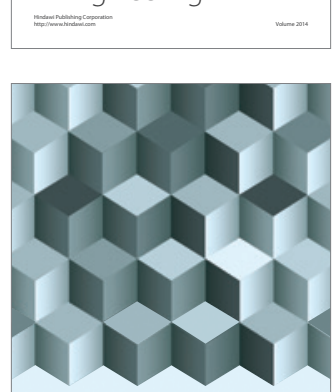

Journal of

Function Spaces
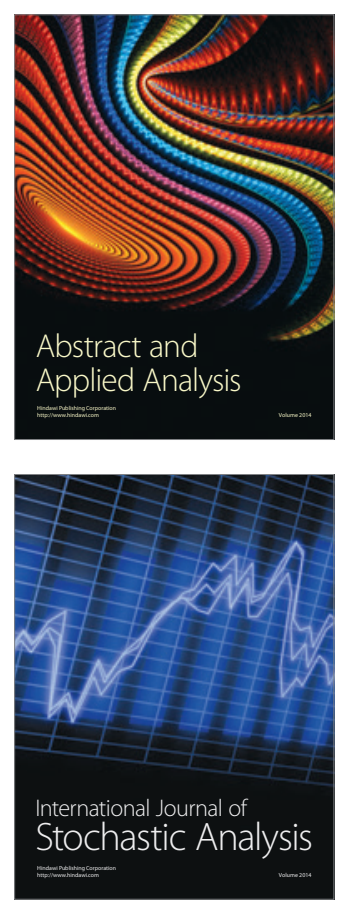

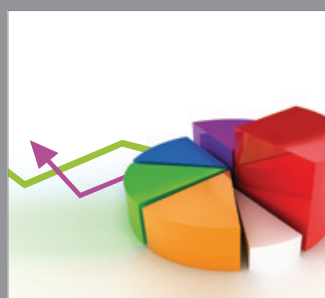

ournal of

Probability and Statistics

Promensencen
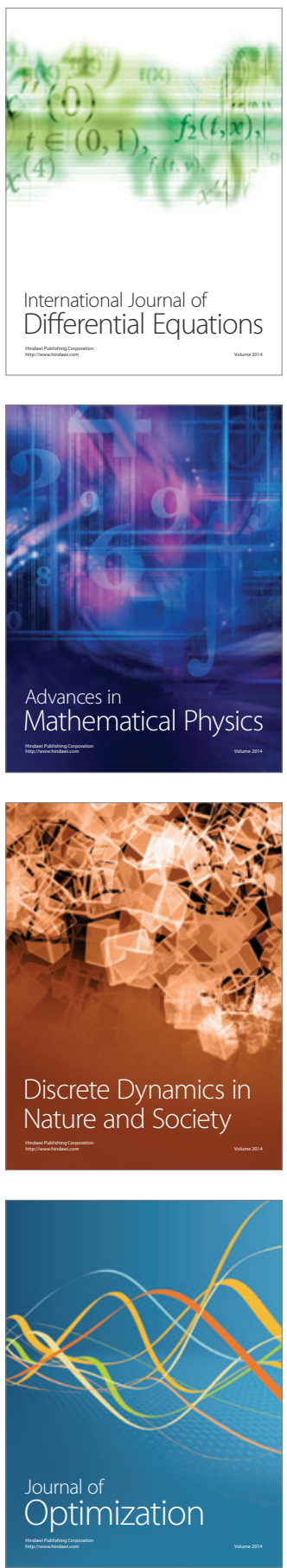